\title{
Quentin Bazin
}

\section{La création brute : altérité et institution}

\section{RAW CREATION: ALTERITY AND INSTITUTION}

Abstract: This paper is an overview of an ongoing investigation on art brut. We will argue that art brut is underpinned by a conceptual imaginary that could lead us to propose alternative cultural mediations. Within art brut, understood as a collection of outsiders' productions, the notion of alterity is always involved, whether it's for its praise, its criticism, its production or its reception. We question the central role of alterity in a four-stage process. The first one introduces the problematical insufficiency of the notion of otherness. The second stage specifies the notion of institution and introduces the hypothesis of a pre-instituted depth. The third stage unfolds this imaginary, whether it concerns the production or the reception of singular forms. The last part intends to show how this theoretical journey could help us to guide practices.

Keywords: Alterity; Imaginary; Creativity; Art brut; Singular; Outsider; Groove; Delirium; Struggle.

\section{QUENTIN BAZIN}

Universités Jean Moulin Lyon III \& Stendhal Grenoble III etrangerleproche@riseup.net
Oet article suit le cheminement d'une thèse en cours de rédaction dont l'enjeu est d'entendre quel imaginaire conceptuel ${ }^{1}$ est sous-jacent à la notion d'art brut, et d'en prendre la mesure pour s'aventurer dans des médiations culturelles originales. Dans la remarquable collection de productions « autres de l'art » qu'est l'art brut, l'altérité (alien ou alter ego, le tout autre ou l'autre familier) est toujours au centre, que ce soit au sein de ses éloges, de ses critiques, de sa production ou de sa réception. C'est la centralité de cette notion qui est ici étudiée et mise en question, à travers quatre étapes. La première introduit l'insuffisance problématique de la notion d'altérité visà-vis de l'art brut, et propose par suite de voir l'altérité comme un faisceau d'opérations spécifiques. La seconde étape précise la notion d'institution, indissociable de celle d'altérité, et présente l'hypothèse d'une profondeur préinstituée. La troisième étape déploie cet imaginaire conceptuel, au niveau de la production ainsi qu'au niveau de la réception de formes autres, singulières. Enfin la dernière étape situe la manière dont ce cheminement théorique est susceptible d'orienter et d'accompagner des pratiques. 


\section{Première étape : une altérité nébuleuse}

$\mathrm{L}$ 'art brut est une notion qui nait sous la plume de l'artiste et écrivain Jean Dubuffet à la fin de la seconde guerre mondiale. Comme tout discours paraissant radicalement nouveau et original, la notion est en réalité, dès ses balbutiements, tissée d'une polyphonie de voix préexistantes, en discussion avec d'autres pratiques : celles du dada, du surréalisme, de la pataphysique, du situationnisme, de médecins psychiatres, de théoriciens de l'art, d'artistes, de collectionneurs, etc. ${ }^{2}$ S'il s'avère difficile de trouver au sein de ses écrits une définition ferme et positive de l'art brut, l'esprit de l'entreprise est de collecter des ouvrages étrangers au monde de l'art et de montrer que la création, dans ce qu'elle a de plus novatrice, se situe davantage dans des milieux « autres " (socialement marginaux, médiumniques et asilaires), que chez les prétendants légitimes aux galeries et musées.

Le rôle que joue l'altérité dans cette description est problématique, et participe à rendre l'art brut nébuleux dans les deux sens de ce terme. Premièrement, l'art brut est « obscur ${ }^{3}$, difficile à comprendre ": l'autre, en débordant des logiques d'identité, échappe aux déterminations hâtives, et pose le problème, concernant les discours ayant pour objet cet autre, d'un sujet énonciateur toujours situé, jamais neutre. Dubuffet est tout entier tendu vers l'autre de l'art et les oubliés de la culture, mais toujours à travers son statut et sa notoriété d'artiste. En travaillant à décrire d'extraordinaires altérités humaines et formelles, il travaillait aussi à sa propre idée de la création. C'est en partie pour cette raison que l'art brut ne trouve pas de définition arrêtée, mais plutôt toutes sortes d'infinitions ${ }^{4}$, des tentatives renouvelées de définitions dont les contours semblent pouvoir varier indéfiniment. Dubuffet était bien conscient de ce flou et n'hésitait pas à l'articuler à la surprenante logique de son discours :

Formuler ce qu'il est cet art brut, sûr que ce n'est pas mon affaire. Définir une chose - or déjà l'isoler c'est l'abîmer beaucoup. C'est la tuer presque [...]. L'art brut, c'est l'art brut et tout le monde a très bien compris. Pas tout à fait très bien ? Bien sûr, c'est pour ça justement qu'on est curieux d'y aller voir. ${ }^{5}$

En second lieu, comme la nébuleuse, l'art brut est « constitué d'amas diffus ». Ses infinitions fonctionnent comme des assemblages de composantes hétérogènes, s'attachant tantôt à décrire la diversité d'origine des œuvres, tantôt leurs différences d'avec l'art professionnel imitatif, leurs qualités formelles ou encore leurs traits remarquables en termes d'inspiration, d'autodidaxie, de clandestinité. L'altérité est ici problématique dans la mesure où par cette seule notion sont amalgamées des réalités singulières, des logiques distinctes. Ces considérations invitent à faire en sorte que l'examen des composantes hétérogènes de l'art brut, à commencer par les milieux de production des œuvres, permette de mieux cerner la notion d'altérité, et de dissiper un peu la confusion inhérente à l'art brut.

L'art brut émerge à une époque où les « arts autres » étaient déjà les objets d'une curiosité renouvelée ${ }^{6}$. Cette notion, inaugurale d'un nouveau rapport à la créativité, a dû s'expliquer et se positionner vis-à-vis 
d'ouvrages de cinq milieux, cinq principales figures d'altérité : les arts primitifs, enfantins, populaires ${ }^{7}$, médiumniques et enfin l'art des fous. Parmi ces cinq figures, seules les trois dernières pourront éventuellement ${ }^{8}$, prétendre au statut d'art brut. Dubuffet juge que la maladresse des enfants n'est pas encore devenue un style singulier et que leur pratique est trop conditionnée par les regards des adultes; tandis que les œuvres des sociétés extra européennes pâtiraient de leur forte appartenance à un monde traditionnel. Si ces deux figures d'altérité échouent à intégrer l'art brut, elles permettent néanmoins d'entendre significativement certains de ses critères. C'est au sein de ces cinq figures hétérogènes que la définition de l'art brut se négocie, se met à prendre consistance, et c'est ainsi depuis l'examen de ces cinq figures que nous entendons approcher une conception originale de la créativité. Mais entre les aliéné.e.s, les membres de sociétés traditionnelles, les enfants, les spirites et les marges populaires, les réalités sont si contrastées que la notion d'altérité en devient suspecte. L'analyse et l'examen de composantes transversales à ces différentes figures d'altérité s'avèrent nécessaires, non pas dans l'optique de trouver ce qu'est l'altérité, mais ce qu'elle occasionne, ou encore les manières qu'elle a de fonctionner ${ }^{9}$.

La première composante repose dans le simple fait que l'altérité est toujours, au sein de ces figures, relationnelle. Les figures d'altérité sont l'autre d'une norme, et constituent en négatif une subjectivité instituée comme normale. Respectivement aux cinq figures d'altérité, cette subjectivité qualifie un sujet adulte, civilisé, un sujet intégré socialement, rationnel, et psychiquement stable. Ainsi, par contraste, la notion d'altérité semble aller de pair avec la constitution, l'institution et la légitimation d'un sujet social et politique standardisé. C'est en ce sens que l'art brut est parfois évoqué comme une convulsion idéologique : en posant à sa manière la question de la part (dans le champ culturel) de celles et ceux qui, refoulé.e.s, nont pas part à ce monde standard.

La seconde composante apparaît en se rapprochant des manières de témoigner, dans les discours, de ces figures d'altérité. L'altérité s'y trouve toujours fabulée, fantasmée, par un genre de projection imaginaire qui institue l'autre en tant qu'autre, en l'intégrant et le traduisant dans un répertoire conventionnel lui-même étranger à l'autre. Les fabulations se reconnaissent par exemple dans leur tension axiologique entre valeur positive et valeur négative de l'altérité : le bon sauvage répond au primitif arriéré, l'innocence enfantine à la perversité infantile, le fou génial au dangereux dégénéré, et ainsi de suite. Cette composante de l'altérité imaginée est par ailleurs exemplaire dans le cas du Douanier Rousseau qui fut poétiquement " exotisé ${ }^{10}$, ou dans le cas de Dubuffet qui fantasme ${ }^{11}$ la démarche de certain.e.s autodidactes, allant parfois jusqu'à idéaliser leur désintéressement sans tenir compte des faits avérés ${ }^{12}$. Il y a enfin, au sein de cette composante, un trait imaginaire positif récurrent : celui selon lequel les figures d'altérité bénéficient d'une certaine "intensité ». Les champs lexicaux de l'instinct, de l'authentique, des pulsions primordiales ou archaïques, de la pureté ou de l'indemne, de l'expression universelle, qui représentent des pentes essentialistes assez courantes chez Dubuffet comme chez les actuel.le.s commentateurs et commentatrices de l'art 
brut, indiquent que ce qui légitimeraient in fine le statut d'autre est une proximité privilégiée avec le vivant, des liens anthropocosmiques moins altérés que ceux de la subjectivité normale.

$\mathrm{La}$ troisième composante concerne enfin le rôle que l'altérité peut jouer au sein d'appareils théoriques, de pratiques artistiques et politiques. Par le contraste évoqué dans la première composante de l'altérité, des possibilités de conflits avec les normes semblent ouvertes, dans lesquelles les figures d'altérité peuvent jouer le rôle de leviers. L'étude de dessins d'aliéné.e.s, a permis, pour des médecins comme Prinzhorn, d'avancer dans la contestation des oppositions maladie/santé et art/nonart, ou dans la résistance aux classifications nosographiques étroites. Dada proteste contre lordre établi, que ce soit celui des mœurs, du langage ou de la guerre, en intégrant à sa protestation des éléments du primitivisme. Le surréalisme, de son côté, manifeste contre l'arbitraire de la réalité aussi bien que contre l'exposition coloniale, en intégrant à sa contestation des productions exécutées par des mediums et des fous. Aujourd'hui, des manifestivités (selon le jargon d'Uzeste) singulières, apparentées à l'art brut, proposent, dans le cadre de la Biennale Hors-Norme à Lyon ou d'Itinéraires Singuliers à Dijon par exemple, des événements engagés, des rencontres inhabituelles questionnant nos rapports à l'altérité, en œuvrant à remettre en cause les rapports habituels à la culture et à ses relais.

Cette étape aura considéré l'altérité du corpus d'émergence de l'art brut à travers trois facettes : l'altérité apparaît moins comme propriété objective que comme mode relationnel qui nourrit les normes, comme opération imaginaire qui donne l'occasion de fabuler, particulièrement à travers l'imaginaire de l'intensité, et enfin comme possible levier permettant d'aviver une transformation des normes. Tout au long de cette description, la notion d'institution est mobilisée explicitement ou implicitement avec les normes instituées, l'institution imaginaire, et la dimension instituante. Le rapport à l'institution tenant également une place centrale dans l'art brut, son sens va être précisé.

\section{Seconde étape :}

\section{altérité et institution}

Q

ue ce soit dans ses pamphlets ou dans sa correspondance, Dubuffet insiste inlassablement sur le rapport contraignant que l'institution joue sur la créativité. L'institution, au premier titre qualifiée d'asphyxiante ${ }^{13}$, y est à prendre au sens large : le langage est une institution, les habitudes cristallisées, les manières d'apprendre (à dessiner par exemple) ou de désirer (être artiste par exemple) en sont d'autres, ainsi que la culture officielle et son lieu privilégié, le musée. L'institution semble être à entendre généralement comme une manière de décrire tout ce qui tend à diriger et à répéter des comportements, des manières de faire, des rapports au monde, c'est-à-dire à produire de la subjectivité. Le langage en tant qu'institution est ce qui oriente notre pensée dans des énonciations types par un répertoire préexistant et dont l'usage nous façonne de manière contagieuse. Le désir d'exposer, d'être reconnu, ou la manière de dessiner relèvent de conséquences de l'institution, où l'institution réside dans l'éducation, la prescription de gestes, mais aussi dans des structures 
prescriptives plus anodines et moins remarquables (des accordages affectifs par exemple). Enfin le musée symbolise l'institution par excellence pour Dubuffet : un lieu clos montrant, sur un mode concentrationnaire ${ }^{14}$, toujours les mêmes formes retenues par la culture, et soumettant les démarches et les voix. En affirmant « l'art ne vient pas coucher dans les lits qu'on a faits pour lui », Dubuffet nous suggère une image qui pense l'institution de manière touchante : le lit, l'ornière, ou mieux encore, le sillon. L'art, dès qu'il s'en tient à suivre un sillon déjà tracé, perd ce qui fait sa spécificité : déborder, frayer de nouvelles voies, de nouvelles formes. Si le flux est la production incessante de formes (scientifiques, ordinaires, politiques, artistiques, etc.), il se trouve qu'à l'instar du langage, de la culture ou de ses lieux, le sillon oriente, dirige, perpétue dans des formes reconduites ce flux.

Certes, la dynamique de l'institution est pour partie asphyxiante, elle a tendance à encoder le processus vivant en identité fixe, la singularité en potentielle convention : une condamnation de l'institution est légitime, tout comme l'est l'idée et la pratique d'un incessant conflit avec elle. Mais l'institution est aussi la condition de constitution de toute pratique et de toute pensée, $\mathrm{y}$ compris celles cherchant à la révolutionner, ou à la subvertir ${ }^{15}$. C'est, dans une certaine mesure, ce à quoi assiste Dubuffet lors de l'un de ses premiers « braconnages " à l'hôpital psychiatrique de Saint-Alban-sur-Limagnoles, en Lozère : la psychothérapie institutionnelle alors en germe décide de travailler à soigner l'institution, et ce avant de prétendre être en mesure de soigner les fous. Le travail institutionnel se développe alors tout contre l'institution qui a déjà montré des signes manifestes de maladie ${ }^{16}$. Malade de guerres absurdes, malade de loccidentale normemâle, malade de la gestion et de la rationalité instrumentale, malade du spectacle et de l'imitation... Une culture, lasse d'ellemême et consciente de son rôle transformateur sur les individus, se met alors à chercher, au sein de l'institution, de nouveaux rapports à ces « autres » de l'institution (principalement les fous et les enfants pour la psychothérapie et la pédagogie institutionnelle), suivant l'idée de Bonnafé selon laquelle « on juge le degré de civilisation d'une société à la manière dont elle traite ses marges, ses fous et ses déviants ». L'autre devient là l'occasion d'une salvatrice remise en question de la compulsion institutionnelle du même et de la norme. Si piégés dans les sillons, la culture instituée nous fait régulièrement persévérer dans des usages du monde ennuyeux et destructeurs, dans le même temps, ces sillons laissent aussi entrevoir leur éventuel débordement : «Une ornière, c'est ce dont il est possible de s'échapper ${ }^{17}$.

L'institution peut donc être pensée par une image issue de l'art brut, et liée avec les mouvements institutionnels contemporains au développement des idées de Dubuffet. Un autre motif, probablement le plus récurrent du champ lexical des écrits sur l'art brut, permet de nourrir l'imaginaire conceptuel qui sous-tend l'art brut : celui de la topologie souterraine. Dès les premières mentions de l'inventivité des dessins de fous, ces derniers sont comparés à des " fossiles précieux et splendides ${ }^{18}$ qui ne doivent leur lointaine remontée qu'à la maladie psychique. Puis à des eaux souterraines rejoignant le fleuve de l'art ${ }^{19}$. L'art brut a historiquement commencé par s'exposer dans une cave, le sous-sol de 
la galerie René Drouin à Paris, puis plus tard des associations filles de l'art brut se sont nommées L'Aracine $e^{20}$, ou Mycelium ${ }^{21}$. Il est enfin difficile de ne pas tomber, chez Dubuffet ou d'autres amateurs d'art brut, sur un recours régulier aux notions de " fond ", de " profondeur ", de " couches sous-jacentes » pour éclairer le mécanisme de créativité propre à l'art brut.

L'articulation de la notion d'institution et du motif de la profondeur permet d'avancer dans la conception de ce mécanisme. L'art brut, lorsqu'on l'entend avec les préoccupations philosophiques de son époque, semble accompagner l'imaginaire suivant : avant la naissance des personnes humaines, celles-ci baignent dans une dimension préinstituée, dans un état en-deçà de toute distinction entre sujet et objet, ou de toute autre détermination possible. Le fotus puis le tout petit humain vivent peu à peu la structuration sensible, la prise de consistance d'un rapport au monde qui s'institue. Plus tard ces institutions qui amendent et corrigent le rapport au monde vont croissantes, et passent par des étapes notables, comme la prise de conscience d'un corps, la contraction d'un langage, d'une subjectivité, etc. À la fois forme et trajectoire de vie, en empruntant ces sillons, la personne humaine fait peu à peu se sédimenter des couches instituées sur ce fond préinstitué. Mais ce fond, bien que recouvert, latent, inobjectivable, s'il n'est pas une substance, nous ne cessons pas d'être en contact avec lui, il est comme ce sur quoi nos expériences prennent forme ${ }^{22}$. Ainsi toute expérience admet une sousjacence, une profondeur préinstituée, qui conditionne notre rapport au monde.

L'imaginaire conceptuel de la créativité que manifeste l'art brut suit l'idée maldinéenne selon laquelle certaines créativités, plutôt que de répondre massivement à un projet du sujet (qui ne se qualifie qu'en des termes institués), répondent davantage à un appel de cette profondeur. De plus, cet appel est davantage audible dans certaines dispositions qu'illustrent les trois corpus majeurs (et mêlés ${ }^{23}$ ) de l'art brut ; les troubles psychotiques, dans lesquels le rapport habituel au monde est en crise, dans lesquels la création est dite vicariante, c'est-à-dire qu'elle tente de "tenir lieu " d'un soi mal assuré24. Les transes médiumniques, dans lesquelles la personne n'a plus à endosser son statut de sujet, puisque le soi y est délégué à des instances étrangères, à des esprits. Et enfin, les situations d'isolement, d'enfermement, de marginalité et de désocialisation, au sein desquelles les parts instituées des personnes ne jouent pas ou plus leur rôle de couche couvrante : leur constitution existentielle s'étant déroulée hors des sillons coutumiers, le fond y serait partiellement à nu. Cet imaginaire à mi-chemin entre esthétique, psychiatrie, sociologie et philosophie devrait être moins entendu dans la vérité de ses termes (que ce soit celui de la profondeur préinstituée d'un côté, d'une existence pleinement instituée de l'autre), que dans l'hétérogénéité du continuum sensible se déployant entre ces pôles, permettant par exemple d'éclairer la présence du fantasme de l'intensité : les œuvres seraient, du point de vue d'un sujet, de moins en moins intenses à mesure qu'elles s'élaboreraient dans des dispositions et des opérations objectives, intentionnelles, balisées et aisément reconnaissables.

La question de cette profondeur préinstituée souffre toutefois d'être évacuée à la manière de la théologie négative (dans 
une description dépourvue de qualificatifs positifs). D'autant que durant la seconde moitié du $\mathrm{XX}^{\mathrm{e}}$ siècle, de nombreuses pensées ont fait l'hypothèse d'une telle entité, à la fois fondamentale et inobjectivable. Ces pensées ont par ailleurs, pour la majorité d'entre elles, fait du problème de la création leur foyer. Nourrissant la question de cette profondeur, il y a les notions de Chaos et de magma chez Castoriadis, de chaos également chez Guattari et Deleuze, qui théorisent aussi une dimension (le moléculaire ${ }^{25}$ ) associée à ce chaos, l'Ouvert chez Maldiney, et enfin le préindividuel chez Simondon. Ces notions trouvent un ancêtre commun chez Anaximandre : l'apeiron (ö $\pi \varepsilon \imath \rho o v)$, qui est une intuition selon laquelle il est à considérer, avant toute autre chose, une indétermination fondamentale, qui est aussi la force d'engendrement, la réserve de devenir de tout être. Mis à part cet ancêtre commun et une relative compatibilité entre ces notions, elles recouvrent des sens différents qui ne seront pas détaillés ici, se liant tantôt avec le vocabulaire scientifique (par la notion de métastabilité), politique (par les questions relatives au déterminisme historique, à l'autonomie), et également phénoménologique (par la notion de pré-langagier, de pathique ${ }^{26}$ ), l'intérêt de ce dernier registre étant d'insister sur la dimension sensible, expérientielle de cette profondeur préinstituée ${ }^{27}$.

L'avancée de cette étape peut être ramassée en quatre points, complétés par l'étude du corpus théorique précédent :

- L'institution, en tant qu'instance permettant la reproduction de comportements, trouve une image évocatrice dans le sillon. Par ailleurs, sous des abords anti-institutionnels, l'art brut gagne davantage à être entendu, dans sa dimension conflictuelle, comme opportunité de transformer et de subvertir les institutions établies.

- Cette puissance de remise en question de l'institué serait issue du commerce qui s'effectue entre les créativités brutes et l'imaginaire d'une profondeur préinstituée progressivement recouvert par des déterminations s'instituant. Les trois corpus privilégiés de l'art brut et le fantasme de l'intensité peuvent être relus à l'aune de fond.

- Parallèlement à cette élaboration métaphorique de Dubuffet, s'échafaudent, à la même époque, des philosophies posant une profondeur préinstituée comparable : un fond à la fois « fond de la vie $»^{28}$, inobjectivable, indéterminé, réserve pour les singularités ${ }^{29}$ et principe de génération de toutes formes.

- Toute forme nouvelle (qu'elle soit « art de faire » quotidien, manière de s'organiser politiquement, invention artistique ou scientifique) est prise de consistance d'une singularité, charriant avec elle des parts préinstituées (les parts brutes), advenant comme autre de l'institution, débordant de l'institution préexistante. C'est l'expérimentation esthétique (au sens grec, aisthesis, de sentir) des trajets et de la remontée de ces parts brutes qui est l'objet de l'étape à venir.

\section{Troisième étape : \\ courts-circuits et ostranénie}

$\mathrm{D}_{\mathrm{s}}^{\mathrm{s}}$ ubuffet souscrivait à l'idée ancienne selon laquelle l'art inventif est toujours conditionné par la folie. Il apparaît aujourd'hui que l'art inventif, mais plus encore toute production de forme nouvelle, toute forme de vie nouvelle, l'est dans la mesure où il y a effectivement une 
part de délire, entendu dans plusieurs sens autour desquels des dissensions gravitent, particulièrement les liens entre souffrance et créativité $^{30}$. Le délire, non-réductible à une catégorie nosographique, admet un sens commun d'exaltation, et un sens inspiré de la psychologie de Freud : le délire est une tentative de guérison, de rendre plus habitable le vécu et le sens d'une expérience particulière ou générale, que le sujet a du mal à élaborer ${ }^{31}$. Or les expériences qui " côtoient » le préinstitué, sont exemplairement susceptibles de mettre en échec la mise en représentation cohérente. C'est même à cette difficile intégration qu'elles sont reconnaissables. Autrement dit, lorsqu'il y a un écart important entre le à dire et les possibilités formelles, instituées de dire (dans ce qui, donc, a déjà été $d i t^{32}$ ), lorsque les mots, les formes, les gestes, les notes, les couleurs manquent, c'est qu'il y a à la fois discernement d'une insuffisance des répertoires institués, et la perspective d'une potentielle percée délirante, hors des sillons déjà frayés. Délire signifiant étymologiquement (delirare) une sortie du sillon, il trouve son troisième sens en se liant à l'imaginaire conceptuel de la créativité. De là, l'invention de formes encore inédites pourrait provenir d'un mélange de ces deux pôles :

- être exposé à des expériences qui " chaotisent " notre rapport institué au monde, et dont des parts brutes, des singularités trouveraient, en s'imposant au sujet, une manière de "remonter " des profondeurs, dans des formes nouvelles. L'auteur.e d'art brut loge dans ce premier pôle, et le plus souvent sa spécificité, en plus d'être étranger au monde de l'art, est d'être à ses dépens dans cette situation délirante, de n’avoir pas recherché une expérience (hallucination, isolement, traumatisme...) en vue de stimuler son inventivité.

- le second consistant à rechercher, à travailler une manière de dire ou de faire permettant d'exprimer un rapport au monde qui ne s'est pas encore institué comme forme. L'artiste n'est plus depuis longtemps le représentant privilégié de ce second cas : sur des modalités propres, des artisans, des chercheurs et des chercheuses, des amoureu.x.ses, des personnes à des postes dits " créatifs ", des individus de tout poil passent par ce travail.

Toutefois, les artistes sont encore reconnus, dans l'imaginaire collectif, comme les ambassadeurs privilégiés du commerce avec les profondeurs. Pour permettre le délire, la remontée de formes nouvelles, ils sont à même d'expérimenter des courts-circuits sur les couches instituées recouvrant le fond et qui empêchent le délire, tout en évitant de s'enfermer dans des troubles psychotiques. Parmi les affections jouant le rôle de courts-circuits, il est possible de citer l'historique et ambigüe mélancolie ${ }^{33}$, celle faisant des artistes les «enfants de Saturne " ${ }^{34}$. Les courts-circuits peuvent également tenter de se formuler physiologiquement, par la production d'endorphines, qui sont sécrétées dans de diverses situations (par exemple dans la perturbation des cycles de l'alimentation ou du sommeil, par des exercices physiques intenses, par les chants, ritournelles et autres mantras accompagnant les gestes, ...) $)^{35}$ attestées dans de nombreuses biographies d'artistes. Enfin, les courts-circuits peuvent apparaître dans des pratiques de désapprentissage plus ordinaires (selon l'adage suivant: « quand je sais dessiner de la main droite, je passe à la main gauche »), ou dans toute technique ménageant une 
place au hasard et à l'indéterminé, délestant l'auteur.e d'une intentionnalité qui aurait l'inconvénient de charrier avec elle de l'institué.

Court-circuiter revient donc à trouver, d'autres manières, inhabituelles, de faire émerger des formes. Il est à noter qu'à travers ces considérations, le risque est toutefois d'enfermer la créativité dans une simple expression, c'est-à-dire de distinguer nettement le «à dire » de la manière de le dire, et conséquemment d'imaginer qu'un « à dire » préexisterait aux manières de le dire. Dans le terme "préinstitué », comme dans celui de « préindividuel », le " pré » est en effet un piège, l'enjeu de ces formules est davantage logique que chronologique. L'expression ne doit pas non plus éclipser le fait que pour prendre forme, les formes ont à négocier physiquement avec la résistance des matériaux spécifiques à l'activité créatrice.

Aux courts-circuits du moment de la création répondent des mécanismes ayant lieu dans le moment de la réception d'une forme nouvelle. Plus haut, dans le quatrième point de la seconde étape, il était proposé que l'altérité puisse produire l'effet d'ouvrir sur une transformation, c'est-à-dire de passer de l'institution en tant qu'elle est déjà réalisée (dimension instituée), à l'institution en tant qu'elle se constitue (dimension instituante). Cet effet transformateur de l'altérité est assez évident dans les expériences ordinaires : la rencontre d'une altérité aura davantage de retentissement dans un comportement que la rencontre de comportements identiques à celui-ci (par exemple, c'est en côtoyant de nouvelles manières de cuisiner que les techniques culinaires, même les vieilles recettes, se mettent à changer).

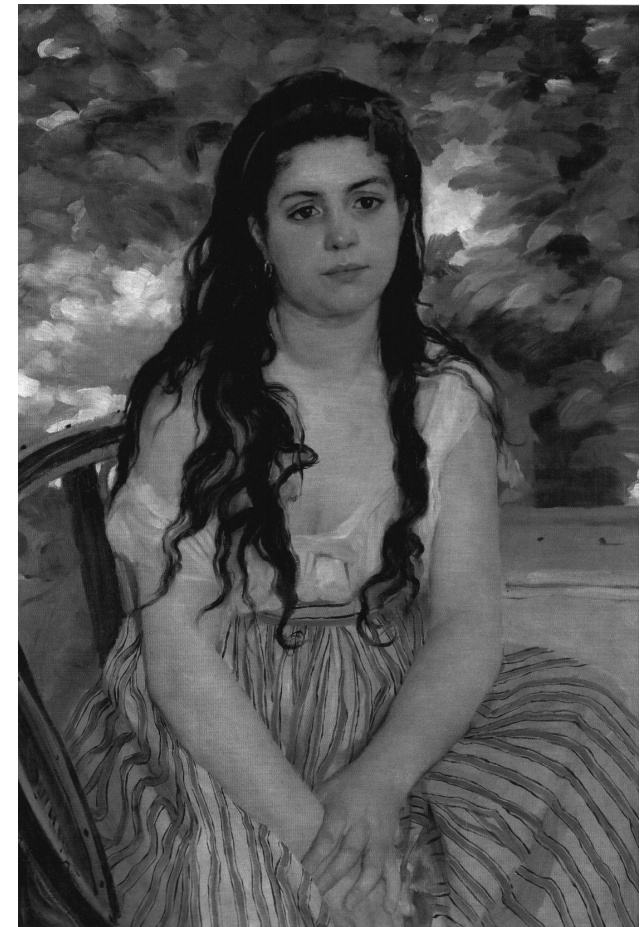

Mais cette portée transformatrice est nettement moins évidente concernant l'expérience esthétique : comment le spectacle de formes nouvelles nous transforme-t-il ? Afin d'essayer d'avancer dans cette question, il y a dans l'art officiel un exemple qui repose dans ce qui se passe entre ces deux toiles de Renoir, et dans le temps qui suit leur réception : En Été datée de 1868 et $L a$ Rêverie, datée de 1877.

(...) le peintre original, l'artiste original procèdent à la façon des oculistes. Le traitement par leur peinture, par leur prose, n'est pas toujours agréable. Quand il est terminé, le praticien nous dit : "Maintenant regardez." Et voici que le monde... nous apparait entièrement différent de l'ancien, mais parfaitement clair. Des femmes passent 


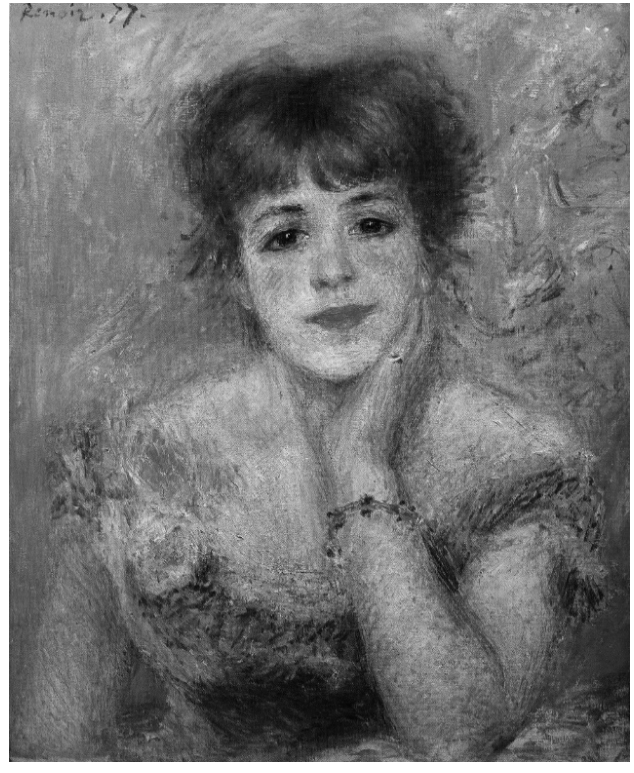

dans la rue, différentes de celles d'autrefois, puisque ce sont des Renoir, ces Renoir où nous nous refusions jadis à voir des femmes. Les voitures aussi sont des Renoir, et l'eau, et le ciel $[\ldots]^{36}$

Tandis que le sujet du premier tableau est aisément reconnaissable - la chair, les cheveux, les tissus étant rendus dans une précision "réaliste " - , la touche du second, plus épaisse et trouble, surtout au niveau de la robe et des bras, le rend plus déstabilisant. Cet écart était naturellement bien plus sensible à la fin du XIX $\mathrm{X}^{\mathrm{e}}$ siècle, puisque depuis cette époque, la représentation, les images impressionnistes sont «passées » dans les sillons communs. Proust indique deux éléments marquants : que la déstabilisation des habitudes allait jusqu'au refus de reconnaître le sujet du tableau (dans la mesure où, explique-t-il, la difficulté à reconnaître n'est pas agréable), et que par la suite, c'est un monde entier qui s'est rafraîchi, substitué à l'ancien - les regards s'artialisent ${ }^{37}$. Même si la transformation esthétique peut paraître exagérée, l'hypothèse selon laquelle les regards sont pétris de schèmes institués (c'est-à-dire que nous avons par exemple, par le biais d'images, de descriptions, une idée assez stable de la mer avant d'y être allé : il y a là un schème de la mer), et que certaines rencontres renouvellent ces schèmes, paraît digne d'être examinée. D'autant plus qu'il apparaît évident que changer le rapport à ces schèmes conditionne et ouvre la possibilité d'agir différemment avec les réalités correspondantes.

Ces considérations permettent de réactiver une théorie plus ancienne, celle que Victor Chklovski introduit dans un ouvrage de 1917 à propos de la littérature, et que nous généralisons ici aux expériences esthétiques. Selon cette théorie, l'expérience esthétique est capable de produire des ostranénies (traduites par défamiliarisation ou étrangéisation), qui se manifestent lorsque les habitudes perceptives (ou les schèmes institués) sont malmenées par une altérité sensible. Ou, autrement dit lorsque les automatismes perceptifs ne suffisent pas pour reconnaître immédiatement une forme (littéraire, musicale, corporelle, plastique, vidéo, etc.). Comme ces formes ne peuvent être reconnues immédiatement, ne peuvent s'assimiler aux formes déjà expérimentées, elles poussent le sujet à voir. Chklovski définit l'art par cette distinction :

Pour rendre la sensation de la vie [...] il existe ce que l'on appelle l'art. Le but de l'art, c'est de donner une sensation de l'objet comme vision et non pas comme reconnaissance ; le procédé de 
l'art est le procédé de singularisation des objets et le procédé qui consiste à obscurcir la forme, à augmenter la difficulté et la durée de la perception. L'acte de perception en art est une fin en soi et doit être prolongé ; l'art est un moyen d'éprouver le devenir de l'objet, ce qui est déjà "devenu" n'importe pas pour l'art. ${ }^{38}$

L'ostranénie s'articule de manière assez cohérente avec l'imaginaire conceptuel de la créativité de la troisième étape, où ce qui est « déjà devenu » renvoie à l'institué, et où le " procédé de singularisation » fait écho aux remontées préinstituées. L'ostranénie permet donc de voir (ou de sentir) d'une manière inédite, c'est-à-dire de sentir un écart avec tout ce qui a pu être senti auparavant. Cet écart est ménagé par quelque chose a priori d'anodin : une nouvelle touche du pinceau, une nouvelle disposition de traits et de couleur, un nouveau visage, d'une nouvelle syntaxe, un nouvel agencement d'image et de son. Et cet écart va produire, chez le sujet, une nouvelle tonalité du sentir, lui faire expérimenter des affects encore restés dans l'angle mort du répertoire institué des formes. $\mathrm{Si}$, pour introduire une nouvelle distinction, reconnaître des formes fait appel à notre perception, et voir la singularité de formes nouvelles (leurs parts brutes, leur forme en tant qu'elle n'est pas encore vraiment formée) mobilise notre sentir, le propre de l'expérience esthétique est que dans celle-ci, " rien ne dégrade le sentir en percevoir ${ }^{39}$. Maldiney inaugure cette distinction en disant : "si, comme l'affirme Erwin Straus, la perception est au sentir ce que le mot est au cri, il nous faut alors reconnaître qu' 'à l'origine de toute œuvre d'art, il y a un cri” ${ }^{40}$.
Pour se décaler d'une vision qui parfois semble perpétuer le privilège de l'art aux dépens des vécus ordinaires, notons que l'ostranénie, revenant en un sens au fait d'éprouver cette distinction, est également accessible par des expériences simples et extra-artistiques, comme par exemple le fait d'insister dans la reconnaissance de son propre reflet dans un miroir, ou dans la répétition prolongée d'un même mot à voix haute. Dans ces deux expériences, il y a bien une augmentation de la durée de la perception, et celle-ci semble suffire à permettre l'irruption d'une altérité profonde. Dans un cas, une inquiétante familiarité nous saisit, un autre nous regarde. Dans l'autre, signifié et signifiant se détachent comme s'ils étaient joints par une colle de mauvaise qualité, le langage apparaissant dans son arbitraire, ni naturel, ni rationnel, et en un sens, ni suffisant.

Les courts-circuits et les ostranénies, qu'il paraît vain de limiter à l'art (et a fortiori à l'art brut), témoignent, sur leurs propres modes, que le rapport institué au monde vacille de temps à autre et laisse un instant entrevoir son fond qui n'est ni objectivable, ni institué, mais saturé de singularités. La créativité cherche à frayer, vis-à-vis de cette profondeur, des expériences de courts-circuits, « de descente et de remontée », nécessairement délirantes, afin de faire émerger des formes dont la singularité n'a pas été totalement épuisée par les sillons institués. C'est l'altérité singulière de ces formes qui provoque l'ostranénie, soit l'expérience esthétique qui s'apparente à une épreuve : sentir, en-deçà de percevoir, la nouveauté radicale de cette forme qui non seulement s'impose aux sens, mais également à notre rapport durable au monde, à notre manière de reconnaître ou de ne pas reconnaître, et qui conditionne in fine toute rencontre. 
L'expérience esthétique débouche sur une production de nouveaux rapports au monde, une production de subjectivité par singularisation plutôt que par normalisation. Nous prenons forme dans et par des expériences et des ouvrages singuliers, qui nous font pousser de nouveaux yeux, de nouvelles oreilles, de nouvelles antennes pour sentir et pratiquer différemment le monde qui nous entoure $^{41}$. La tension qui se joue entre l'institué et le singulier, transversale à toutes les étapes de cet article, s'ouvre maintenant sur le champ des questions attachées à notre orientation dans les institutions contemporaines, et dans les motifs qui peuvent guider nos différentes prises de formes.

\section{Quatrième étape :}

\section{un imaginaire conceptuel en lutte}

工 'imaginaire conceptuel de la créativité que nous avons décrit n'est pas une construction anhistorique mais est d'emblée branché sur l'actualité des institutions ainsi que sur les situations et les subjectivités concrètes qu'elles produisent. En effet :

- si l'esthétique n'est pas une théorie de l'art institué mais un opérateur de transformation et de singularisation de la subjectivité, une possibilisation de nouveaux rapports au monde, ainsi que les notions d'ostranénie et de courts-circuits de l'étape précédente l'argumentent ;

- si la dimension de conflit vis-à-vis de l'institution, de travail tout contre l'institution est d'autant plus sensé et requis que les mécanismes d'assujettissement et de normopathie $^{42}$ se doublent actuellement d'une perspective d'effondrement généralisé, potentialisé par les inégalités sociales, les crises environnementales, les politiques ultralibérales ou identitaires ;
- alors il n'est pas étonnant que cet imaginaire conceptuel, chez certain.e.s auteur.e.s, devienne également un imaginaire en lutte, qui se traduit dans des attitudes, des tactiques, une praxis.

Cette praxis se présente dans le déploiement du troisième sens $\mathrm{du}$ mot sens : Sens et perception (renvoyant au sentir, et à l'étape précédente), Sens et signification (renvoyant à l'intelligibilité, et à la quatrième note de ce texte), et enfin Sens et orientation, renvoyant au fait que ces distinctions (entre sentir et percevoir, et entre sens et signification) font vaciller les institutions et les font apparaître telles qu'elles sont : ni rationnelles, ni naturelles, mais arbitraires, et fréquemment défectueuses. Elles peuvent donc être autrement, elles deviennent dignes de défiance, d'où l'envie de les orienter dans d'autres directions, et nous avec elles.

Au sein de ce travail, cette praxis se traduit dans des propositions de médiation culturelle qui sont animées par la question de la reproduction (ou de la mise en crise) des modes de subjectivation dominants. C'est-à-dire de s'orienter parmi des sillons majoritaires qui engourdissent, des débordements joyeux dans les lits mineurs, des voies délirantes partiellement frayées, et parmi toutes celles encore qui restent à ouvrir. Les propositions gravitent autour d'un circuit en AuvergneRhône-Alpes, d'une caravane d'ateliers sillonnant ce circuit, et d'un lieu urbain associatif ouvert au public. Le circuit de curiosités se fera entre des installations visionnaires de la région et des lieux d'art brut, populaire, singulier (entre autres le Jardin Rosa Mir, l'Ermitage du mont Cindre, le Petit Musée de Pierre Martelanche, le Palais Idéal, et de nombreux 
autres lieux ${ }^{43}$, où chaque site pourra faire signe vers ceux voisins. Afin que ce circuit aille à la rencontre des habitant.e.s, une caravane de curiosités populaires proposera, en réponse à la statique des musées, des projections et des ateliers ${ }^{44}$ itinérants faisant connaître des autodidactes locaux ainsi que d'autres du monde entier. Enfin, il s'agira de travailler avec l'association La Sauce singulière, qui organise depuis 2005 les Biennales internationales Hors
Normes en Rhône-Alpes, pour investir un espace afin d'accompagner au mieux les publics et les non-publics ${ }^{45}$ dans ces rencontres, qui chaque année se tiennent dans toujours plus de lieux de vie désertés par les cultures financées. Ces dernières sont progressivement à remplacer par des cultures autres, dynamisant les imaginaires par des délires singuliers, et permettant de « bricoler des zones d'habitabilité " ${ }^{46}$, communes, un peu partout.

\section{BibLIOGRAPHIE}

Pascale Ancel, Alain Pessin (dir.), Les non-publics. Les arts en réception. Paris, L'Harmattan, 2004.

Georges Bataille, « Informe », in Documents, 7, 1929.

Guillaume Le Blanc, L’insurrection des vies minuscules, Montrouge, Bayard, 2014.

Victor Chklovski, «L'Art comme procédé », in Tzvetan Todorov, Théorie de la littérature, Paris, Le Seuil, 1965.

Jean Dubuffet, Prospectus et tous écrits suivants, t. I, Paris, Gallimard, 1967.

Jean Dubuffet, Prospectus et tous écrits suivants, t. II, Paris, Gallimard, 1967.

Jean Dubuffet, Prospectus et tous écrits suivants, t. III, Paris, Gallimard, 1995.

Françoise Dastur, « Henri Maldiney Une phénoménologie de la rencontre et de l'événement », in L'Ouvert, $\mathrm{n}^{\circ} 1,2008$.

Céline Delavaux, L'Art brut, un fantasme de peintre: Jean Dubuffet et les enjeux d'un discours, Paris, Palette, 2010.

Didier Debaise, Isabelle Stengers (dir.), Gestes spéculatifs, Dijon, Les Presses du réel, 2015.

Didier Debaise, «Qu'est-ce qu'une pensée relationnelle ? », in Multitudes, vol. 18, no. 4, 2004.

Sigmund Freud, Le président Schreber, Un cas de paranoïa, Paris, Payot-Rivages, 2011.

Félix Guattari, Suely Rolnik, Micropolitiques, Paris, Les Empêcheurs de tourner en rond, 2007.

Bin Kimura, L’Entre, Une approche phénoménologique de la schizophrénie, Grenoble, Éditions Jérôme Millon, 2000.

Bruno Montpied, Le gazouillis des éléphants, Paris, Éditions du Sandre, 2017.

Jean Oury, Création et Schizophrénie, Paris, Galilée, 1989.

Hans Prinzhorn, Expressions de la folie : dessins, peintures, sculptures d'asile, Paris, Gallimard, 1984.

Marcel Proust, Du Côté de Guermantes, À la recherche du temps perdu, t. II, Paris, Gallimard, 1969.

Benjamin Rush, An inquiry into the effects of ardent spirits upon the human body and mind, Boston, James Loring, 1819.

Jean Starobinski, L'encre de la mélancolie, Paris, Points, 2015.

Erwin Straus, Du sens des sens, Grenoble, Éditions Jérôme Million, 2000.

Carole Talon, « La dimension affective du sentir dans l'expérience esthétique », in Philosophique, $\mathrm{n}^{\circ} 2$, 1999.

Michel Thévoz, Requiem pour la folie, Paris, La Différence, 2017.

David Vercauteren, Micropolitiques des groupes, Paris, Les Prairies Ordinaires, 2011.

Margot et Rudolf Wittkower, Les Enfants de Saturne. Psychologie et comportement des artistes, de l'Antiquité à la Révolution française, Paris, Macula, 1985. 


\section{Notes}

1. Un imaginaire conceptuel désigne une tentative de comprendre un phénomène, souvent complexe et difficilement exprimable (ici, celui de la créativité), par le biais d'une élaboration (mentale ou matérielle) associant des images évocatrices et des concepts ou des notions.

2. La correspondance de Dubuffet atteste de quantité de contacts, d'échanges, d'emprunts directs ou indirects aux pensées ou aux pratiques de Tzara, Jorn, Breton, Oury, Prinzhorn, Queneau, Freinet, Bachelard, Ponge, Artaud, Éluard, Paulhan, Chaissac, Bataille, par exemple.

3. Dans une lettre adressée en août 1945 à celui qui lui avait soumis le terme « art obscur ", le peintre suisse René Auberjonois, Dubuffet explique sa préférence pour le terme d'« art brut » (Jean Dubuffet, Prospectus et tous écrits suivants, t. II, Paris, Gallimard, 1967, p. 240).

4. Rejoignant l'intuition de Bataille selon laquelle «Un dictionnaire commencerait à partir du moment où il ne donnerait plus le sens mais les besognes des mots » (Georges Bataille, «Informe », in Documents, 7,1929, p. 382), l'écriture de Dubuffet est préoccupée par la transformation qu'opère le langage sur le rapport au monde. « On ne se méfie pas qu'une chose, quand on la nomme, ça la roussit comme un coup de soleil. » (Jean Dubuffet, Prospectus et tous écrits suivants, t. II, p. 68). À partir de ces considérations peut se dessiner une distinction entre le sens, qui correspond à la dynamique et au travail permanent de conférer une intelligibilité à l'expérience, et la signification, qui est l'assignation d'une intelligibilité déterminée.

5. Jean Dubuffet, Prospectus et tous écrits suivants, t. II, p. 176.

6. Par exemple à travers la poursuite des travaux sur les fous littéraires de Queneau, les ouvrages médiumniques déjà collectionnés par Breton ou les essais de médecins (Morgenthaler, Réja, Prinzhorn, etc.) fascinés par les productions de leurs patients.

7. Les arts populaires décrivent une vaste famille regroupant des productions locales traditionnelles, mais également des productions socialement isolées, en milieu rural, en institutions de détention, etc.

8. Il y a bien sûr des réserves, et au premier titre le fait que parmi ces corpus, seules les productions apparaissant à Dubuffet particulièrement inventives sont retenues. Par ailleurs, un art populaire naïf ne pourra pas être considéré comme brut, dans la mesure où dans l'art naïf il y a une reconduction de thématiques coutumières, ainsi qu'un désir d'accéder à une reconnaissance artistique.

9. Préférer une certaine pragmatique à l'essentialisme peut être vu comme une volonté de prendre au sérieux l'avertissement de Brigitte Fontaine : «Les enfants, le dix-neuvième siècle est terminé. ».

10. Ce qui apparaît bien dans la communication de Laurence Campa lors du colloque «Inventer le douanier Rousseau » qui s'est tenu les 11 et 12 mai 2016 au Musée d'Orsay, et dont l'intervention s'intitulait " Phénomène ou légende ? L'invention poétique du Douanier Rousseau ».

11. Le terme de fantasme fait ici référence à l'ouvrage de Céline Delavaux, qui opère une remarquable approche de l'art brut par les discours : L'Art brut, un fantasme de peintre : Jean Dubuffet et les enjeux d'un discours, Paris, Palette, 2010.

12. Comme le rapportait Pauline Goutain, auteure d'une thèse sur la matérialité des œuvres brutes, au colloque «Actualité et enjeux critiques de l'Art Brut » qui s'est tenu les 3 et 4 novembre 2016 à l'UNIL de Lausanne, au sujet d'Auguste Forestier. Indifférent à tout commerce selon Dubuffet, Forestier fabriquait des statuettes et des jouets en matériaux récupérés qu'il échangeait ensuite contre des cigarettes, du vin ou du chocolat aux paysans de Saint-Alban-sur-Limagnoles de passage devant les portes de l'hôpital psychiatrique dans lequel il était patient.

13. Le terme d'asphyxiante culture, titre d'un pamphlet de Dubuffet, dénonce en effet la part instituée de la culture : «Quand ensuite la culture s'institutionnalise et vient à se confondre avec l'esprit de Sorbonne, les gens ne sont plus du tout conviés à un exercice personnel, mais tout au contraire à seulement répéter littéralement, comme les élèves caporaux le manuel militaire, un code impératif d'orthodoxie. » (Jean Dubuffet, Prospectus et tous écrits suivants, t. III, Paris, Gallimard, 1995, p. 43).

14. Les liens entre hôpitaux psychiatriques et musées pourraient faire l'objet de multiples rapprochements : des individus concentrés, coupés de leur quotidien, assignés à un diagnostic de spécialiste, une nomenclature, etc. 
15. « La culture s'identifie à l'institutionnalisation. [...] L'institutionnalisation est - quelles que soient les positions qui en sont l'objet - ce qu'il faut sans répit combattre, car elle est la force opposée à celle de la pensée individuelle et donc de la vie même; elle est proprement la force contre laquelle la pensée se constitue; elle est à la pensée comme la pesanteur au sauteur, au projectile. » (Jean Dubuffet, Prospectus et tous écrits suivants, t. III, p. 68).

16. Notamment à travers la distinction entre statut, rôle et fonction, l'attention au champ micropolitique ainsi qu'aux ambiances et aux groupes. Voir notamment l'excellent livre (en ligne) de David Vercauteren, Micropolitiques des groupes, Paris, Les Prairies Ordinaires, 2011. Il s'agit par exemple de voir que des rapports à l'institution gagnent à être pensés comme des outils pour la construction de sillons en lutte contre les oppressions (spécisme, sexisme, racisme, âgisme, validisme) et les inégalités sociales.

17. Didier Debaise et Isabelle Stengers (dir.), Gestes Spéculatifs, Dijon, Les Presses du Réel, 2015, p. 7. S'échapper vers où ? Et avec le regard davantage tourné du côté des sillons à éviter, ou de celui du sillage que nous laissons?

18. Benjamin Rush, An inquiry into the effects of ardent spirits upon the human body and mind, Boston, James Loring, 1819, p. 154 (ouvrage en ligne, c'est nous qui traduisons).

19. Hans Prinzhorn, Expressions de la folie : dessins, peintures, sculptures d'asile, Paris, Gallimard, 1984, p. 70.

20. La collection de l'Aracine est visible au Musée Lille Métropole de Villeneuve-d'Ascq.

21. Le mycélium est le réseau souterrain des champignons. Représentant une impressionnante part de la biomasse souterraine, multiplicité acentrée et processuelle, le mycélium est une métaphore privilégiée pour la créativité, et correspond assez à la notion de rhizome de Deleuze et Guattari (contrairement au rhizome lui-même, qui en botanique a grossièrement les caractéristiques d'un tronc couché).

22. L'approche des modalités de ce contact, relevant à la fois de la métaphysique et de la phénoménologie existentielle, est précisée dans la note 27 .

23. Ces corpus nétant pas indépendants : nous savons par exemple que l'exclusion sociale augmente le risque de schizophrénie.

24. Pour plus de détails sur la vicariance, voir Jean Oury, Création et Schizophrénie, Paris, Galilée, 1989, p. 96 et p. 131.

25. Le moléculaire, « opposé » au molaire, désigne les situations en tant qu'elles sont composées d'éléments singuliers, sans que ceux-ci se rapportent à des logiques d'ensembles ou d'identités. Pour davantage de détails, voir Félix Guattari et Suely Rolnik, Micropolitiques, Paris, Les Empêcheurs de tourner en rond, 2007, p. 179-190.

26. Le pathique dit la capacité affective à accueillir et être éprouvé par l'événement. Maldiney reprend ce terme de la phénoménologie psychiatrique de Weizsäcker, puis de Straus.

27. La profondeur préinstituée étant une dimension au sein de laquelle se déroulent nos expériences, il existe une grande diversité de situations, cherchées ou subies, interstitielles, défamiliarisant ou court-circuitant les couches instituées. Parmi celles-ci, la phénoménologie propose la suspension de l'évidence naturelle qui s'est instituée dans notre rapport au monde, pour approcher la sous-jacence originaire de l'expérience, soit le fait que la conscience est constituante plutôt que distincte de la réalité environnante. Sur d'autres modes qu'il reste à décrire attentivement, les rêves, les expériences extraordinaires, les EMC, les épisodes psychotiques, les côtoiements de vertiges affectifs et d'indifférenciation, les chaosmoses de Guattari, sont autant de modalités de présence et d'expériences par lesquelles la profondeur se met un instant à consister. Cette profondeur, approchée seulement par rapports et expériences singulières, ne se laisse jamais dire dans des notions de pureté ou d'universalité ; les institutions court-circuitées ou suspendues conditionnant invariablement ces rapports.

28. "Ce fond, qui génère toute vie dans son activité et qui dépasse la mort de lêtre particulier, est ce à quoi nous nous rapportons sans cesse bien qu'il nous soit impossible de l'objectiver. "Bin Kimura, L'Entre, Une approche phénoménologique de la schizophrénie, Grenoble, Jérôme Million, 2000, p. 23.

29. "Le propre d'une singularité c'est qu'on ne peut pas en définir les effets avant qu'ils ne sétablissent, qu'on ne peut a priori délimiter le territoire dans lequel s'opèreront ses effets » Didier Debaise, "Qu'est-ce qu'une pensée relationnelle ? ", in Multitudes, vol. 18, no. 4, 2004, p. 15-23. 
30. Des spécialistes de l'art brut expriment des points de vue radicaux sur cette question, notamment le fait que les crises, les inconforts psychiques autant que matériels profitent à l'inventivité. Ou bien que la création entretient et prolonge le potentiel psychotique, plutôt que l'apaiser. Un des problèmes de ces propositions est le manque de nuances : les créativités et délires, insuffisamment définies, sont trop hétérogènes pour vraiment coller à ces généralités.

31. Sigmund Freud, Le président Schreber, Un cas de paranoïa, Paris, Payot-Rivages, 2011, p. 139.

32. Selon une autre distinction que faisait Maldiney.

33. Jean Starobinski, L'encre de la mélancolie, Paris, Points, 2015.

34. Margot et Rudolf Wittkower, Les Enfants de Saturne. Psychologie et comportement des artistes, de l'Antiquité à la Révolution française, Paris, Macula, 1985.

35. Cette hypothèse selon laquelle les artistes produiraient de manière autonome, par ces opioïdes endogènes, leurs états modifiés de conscience afin de stimuler leur créativité, est présente dans le chapitre "Le trip, voyage organisé », de l'ouvrage de Michel Thévoz, Requiem pour la folie, Paris, La Différence, 2017.

36. Marcel Proust, Du Côté de Guermantes, À la recherche du temps perdu, t. II, Paris, Gallimard, 1969, p. 623.

37. Alain Roger parle, à propos de ce même exemple, d'une artialisation du regard.

38. Victor Chklovski, "L'Art comme procédé », in Tzvetan Todorov, Théorie de la littérature, Paris, Le Seuil, 1965 , p. 83.

39. Carole Talon, «La dimension affective du sentir dans l'expérience esthétique », in Philosophique, $\mathrm{n}^{\circ} 2$, 1999, p. 15-28.

40. Cité par Françoise Dastur, « Henri Maldiney Une phénoménologie de la rencontre et de l'événement », in L'Ouvert, n¹, 2008, p. 61-71. En réalité, dans Erwin Straus, Du sens des sens, Grenoble, Jérôme Millon, 2000, p. 503, on ne trouve pas « percevoir » mais « connaître ».

41. "Il faudrait que tous les docteurs fassent le grand hara-kiri de l'intelligence, le grand saut dans l'imbécillité extralucide, c'est alors seulement que ça leur pousserait, les millions d'yeux. » (Jean Dubuffet, Prospectus et tous écrits suivants, t. I, p. 199.)

42. Merci à Samuel Thoma pour la source de la notion de normopathie : probablement chez Joyce McDougall, qui l'utilise dans Plaidoyer pour une certaine anormalité, Paris, Gallimard, 1978, pour caractériser le rapport à la fois normatif et pathologique de psychanalystes concernant les sexualités de leurs patient.e.s. Plus tard le mot se réfère à une tendance à se croire normal, à adhérer aux normes (" parce qu'il faut bien »), à se fondre dans les sillons les plus courus, à l'opposé des délires singuliers. Nos institutions étant saturées de normes, les sujets qu'elles produisent en sont logiquement modelés.

43. Le plus complet des inventaires des sites singuliers se trouve dans le magnifique Bruno Montpied, Gazouillis des éléphants, Paris, Éditions du Sandre, 2017.

44. Ateliers à développer ou à rejoindre pour transmettre et expérimenter certains fruits de ce travail sur l'imaginaire conceptuel que permet d'entendre l'art brut. Outre les biographies captivantes à partager, la notion de Talvère permet de parler d'une profondeur préinstituée sans jargon ni longueur : la talvère désigne en occitan la partie du champ non labourée, non sillonnée (interstices, coins inaccessibles, zones de manoeuvre), qui est un reste irréductible mais qui préserve localement la biodiversité sauvage et bénéficie ainsi paradoxalement aux parties cultivées. De là, imaginer, qu'en nous, et débordant de nous, chacun abrite - ou est abrité par - une talvère. Une talvère comme une dimension de l'expérience, vers laquelle on se dirige parfois sensiblement, par exemple dans les rêves et rêveries. L'atelier pourra relier à l'image de la talvère les courts-circuits et les motifs fondamentaux de l'art brut (les existentiaux, que peuvent être les répétitions, saturations, inventaires, enchevêtrements, mythologies biographiques, ...). Les courts-circuits tentent de s'en approcher quand les existentiaux tentent de la décrire, en des polarités générales (l'ouvert et le fermé, le plein et le vide, l'ordonné et le mélangé, le dissocié et le mêlé, l'expliqué et l'inexpliqué, etc.). 45. D'après Pascale Ancel et Alain Pessin, Les non-publics : les arts en réception, vol. 1 et 2, Paris, L'Harmattan, 2004.

46. Guillaume Le Blanc, L’insurrection des vies minuscules, Montrouge, Bayard, 2014, p. 66. 